\title{
Statistical Inference Method for Liner Impedance Eduction with a Shear Grazing Flow
}

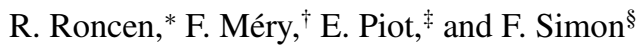 \\ ONERA-The French Aerospace Lab, Université de Toulouse, F-31000 Toulouse, France
}

DOI: $\underline{10.2514 / 1 . J 057559}$

\begin{abstract}
This paper presents a statistical inference method for impedance eduction in a flow-duct facility. The acoustic impedance is recast into a random variable, and Bayes's theorem is used to obtain the posterior probability density function of both its real and imaginary parts, thus expressing the knowledge/uncertainty one has on the impedance value, given a certain experimental data uncertainty. An evolutionary Markov chain Monte Carlo technique is selected to explore the probability space, and a surrogate model based on the method of snapshots is employed to speed up the calculations. The linearized Euler equations are solved using a two-dimensional discontinuous Galerkin scheme, accounting for the presence of a grazing flow. The inference process is first validated on published NASA Grazing Incidence Tube results, in which acoustic-pressure measurements on the wall opposite the liner are used as inputs. Then, the same procedure is applied to educe the impedance of a conventional single degree-of-freedom liner in the ONERA-The French Aerospace Lab B2A acoustic bench, in which a laser Doppler velocimetry (LDV) technique is used to measure the two components of the acoustic-velocity fields above the liner. The primary conclusion of the study is that the Bayesian inference method allows for consistent impedance eductions, as compared to a classical deterministic eduction approach, for both microphone and LDV measurements. Furthermore, it yields the credibility intervals of the identified impedance, which represent the uncertainty on the identified impedance values, given an uncertain measurement. The identified parameters are less correlated using an LDV-based inference than a microphone-based inference, which might be due to the more limited number of data.
\end{abstract}

\begin{tabular}{|c|c|c|}
\hline \multicolumn{3}{|r|}{ Nomenclature } \\
\hline$\left(b_{u}, b_{v}\right)$ & $=$ & axial and vertical measurement noise \\
\hline $\mathcal{C}, \theta_{\mathcal{C}}$ & $=$ & source coefficient and source phase \\
\hline$f, \omega, t$ & $=$ & $\begin{array}{l}\text { driving frequency, circular harmonic frequency } \\
(2 \pi f) \text {, and physical time }\end{array}$ \\
\hline $\boldsymbol{G}$ & $=$ & deterministic forward operator \\
\hline & $=$ & unit imaginary number $(\sqrt{-1})$ \\
\hline$L_{\text {liner }}, W, H$ & $=$ & $\begin{array}{l}\text { total length and width of the liner sample, and } \\
\text { inner height of the liner cavity }\end{array}$ \\
\hline$M_{b}$ & $=$ & bulk flow Mach number \\
\hline $\boldsymbol{m}, \boldsymbol{d}^{\exp }$ & $=$ & model parameters and observable parameters \\
\hline$N_{\text {chains }}, N_{\text {eval }}$ & $=$ & $\begin{array}{l}\text { number of chains used in the Markov chain } \\
\text { Monte Carlo strategy and number of rows in the } \\
\text { posterior matrix }\end{array}$ \\
\hline$n_{m}, n_{d}$ & $=$ & $\begin{array}{l}\text { dimension of parameter space and number of } \\
\text { measurement points }\end{array}$ \\
\hline$p, u, v, \rho$ & $=$ & $\begin{array}{l}\text { acoustic pressure, axial acoustic particle velocity, } \\
\text { transverse acoustic particle velocity, and acoustic } \\
\text { density }\end{array}$ \\
\hline$(U, V)$ & $=$ & 1 mean velocities \\
\hline$(\tilde{U}, \tilde{V}, \tilde{W})$ & $=$ & cal, and spanwise velocities \\
\hline$\left(u^{\prime}, v^{\prime}\right)$ & $=$ & axial an \\
\hline$(x, y, z)$ & $=$ & IWIse cooran \\
\hline$\delta, t_{\mathrm{pp}}$ & $=$ & $\begin{array}{l}\text { liner perforated-plate hole diameter and } \\
\text { thickness }\end{array}$ \\
\hline
\end{tabular}

Presented as Paper 2018-3775 at the 2018 AIAA AVIATION Forum, Atlanta, GA, 25-29 June 2018; received 30 May 2018; revision received 31 August 2018; accepted for publication 18 October 2018; published online 17 December 2018. Copyright (C) 2018 by the American Institute of Aeronautics and Astronautics, Inc. All rights reserved. All requests for copying and permission to reprint should be submitted to CCC at www.copyright.com; employ the ISSN 0001-1452 (print) or 1533-385X (online) to initiate your request. See also AIAA Rights and Permissions www.aiaa.org/randp.

*Ph.D. Student, DMPE, 2 Avenue Edouard Belin; remi.roncen @ onera.fr.

†Research Scientist, DMPE, 2 Avenue Edouard Belin; fabien.mery@ onera.fr.

*Research Scientist, DMPE, 2 Avenue Edouard Belin; estelle.piot@ onera.fr.

${ }^{\S}$ Research Scientist, DMPE, 2 Avenue Edouard Belin; frank.simon@ onera.fr.

\section{Superscripts}

$\begin{array}{ll}\text { exp, num } & =\text { experimental signal and numerical signal } \\ T & =\text { transpose }\end{array}$

\section{Introduction}

$\mathbf{U}$ NDERSTANDING the effects of a complex flow on the acoustical impedance of nacelle liners is of prime importance for current nacelle liner design and has been the focus of many research studies in the past $[\underline{1}, \underline{2}]$.

Two main impedance-eduction philosophies have been put forward in the past: straightforward and indirect methods. Straightforward methods use a measurement of an axial propagation constant in conjunction with the impedance boundary condition to educe the impedance directly, without iterations. Recent examples of developments in this field are found in Refs. [근 -5 . Indirect eduction methods [ $6-8]$ are now a widely spread technique used to retrieve the acoustic impedance of liner materials with indirect nonintrusive measurements, in the presence of a grazing flow. These methods usually consist in measuring the acoustic pressure on the wall opposite the liner and using a minimization method, in which the fields obtained by solving partial differential equations (PDEs) coupled with an impedance boundary condition are compared with experimental measurements. In Jones et al. []], a benchmark for impedance eduction was realised, following this technique. A twodimensional (2-D) and three-dimensional (3-D) finite element method was used to solve the convected Helmholtz equations (CHEs), with a uniform flow hypothesis and an Ingard-Myers impedance boundary condition. Shear flow can be taken into account with other tools based on the Pridmore-Brown equations [9]. 
Many other teams developed eduction methods, all of them based on pressure measurements $[4,10,11]$.

In an attempt to get information closer to the liner than with microphone measurements located on the wall opposite the liner, ONERA-The French Aerospace Lab (ONERA) applied a laser Doppler velocimetry (LDV) technique to measure the two components of the acoustic-velocity fields in the area above the liner, and used them as inputs for the eduction process $[8,12,13]$. The numerical solution of the linearized Euler equations (LEEs) was obtained with a discontinuous Galerkin (DG) scheme, accounting naturally for the presence of a shear flow in the simulation.

Only a few studies have considered the issue of impedanceeduction uncertainty and accuracy. In Brown et al. [14], the authors performed a Monte Carlo sensitivity analysis to study the influence of the Mach number, static temperature, and pressure on the impedance-eduction process. They showed the relative insensitivity of these parameters to the eduction outcome. Later, Zhou and Bodén [15] followed a modal approach, in which the flow was assumed uniform and an Ingard-Myers boundary condition was used at the liner section. The impedance was estimated from the knowledge of the axial wave number, and the uncertainty analysis was carried out on the Mach number. This showed that their impedance-eduction technique was more sensitive to a Machnumber uncertainty at frequencies higher than the antiresonance. Pioneering the use of a Bayesian inference method in impedance eduction, Buot de l'Epine et al. [16] considered a random uncertainty in the synthetically generated acoustic-pressure measurements opposite the liner, and quantified the resulting uncertainties on the identified impedance, in a no-flow case. The impedance was fitted using a model in the frequency domain with seven parameters, which became the parameters of interest during the inference process.

We draw from the previous studies that uncertainty quantifications in impedance eductions are of prime interest, and that the Bayesian inference method allows one to propagate the experimental uncertainty onto the educed impedance, yielding a more general summary of our knowledge regarding the impedance values. However, no such method has yet been performed, to the authors' best knowledge, in the case in which a shear grazing flow is considered.

Compared to previous studies, the present study used a Bayesian inference approach for impedance eduction in the presence of a grazing flow, and the impedance was educed at each frequency. Furthermore, the flow duct was not assumed reflection free at its termination, and the termination impedance was educed as well. Because the acoustic source amplitude and phase were also unknown, the eduction process took these parameters into account as well, totaling a number of six parameters to identify. To accelerate the calculations, a surrogate model based on the method of snapshots was used. The data used as input for the eduction method can be either acoustic-pressure or acoustic-velocity measurements. The acousticvelocity fields were measured by LDV and an advanced postprocessing technique [17] was applied, which also provided the measurement uncertainties. An evolutionary Markov chain Monte Carlo (MCMC) technique was selected to explore the target posterior probability density functions (PDF) of interest.
The outline of the paper is as follows. First, the statistical impedance-eduction strategy is detailed in Sec. II, with an emphasis on the Bayesian inference method, and a presentation of the surrogate model used to accelerate the calculations. In Sec. III, the inference is performed on a data set obtained by NASA on a ceramic tubular liner. This allows the comparison between classical and statistical inference methods. Then, in Sec. IV, ONERA's B2A aeroacoustic bench is presented, along with the LDV measurement and postprocessing used to acquire the velocity fields above the liner. The LDV enhanced measurements are then used to identify the impedance of a conventional single degree-of-freedom (SDOF) liner, and the associated degree of knowledge/uncertainty on the impedance values is summarized. Concluding remarks are laid out in Sec. V.

\section{Statistical Impedance-Eduction Strategy}

This section presents multiple key elements necessary to the impedance-eduction strategy. First, the general geometry of a typical aeroacoustic bench used for impedance eduction is recalled. Then, the direct problem, consisting in solving the LEE, is introduced. Finally, the Bayesian inference method used in this work is detailed.

\section{A. Experimental Setup}

To understand the effects of a complex flow on the impedance of a nacelle liner, a simplified geometry is often considered, as sketched in Fig. 1. Different flow ducts are used around the world for liner eduction $[4, \underline{6}, \underline{8}, \underline{10}]$, but the concepts remain quite similar. Both grazing flow and acoustic plane waves are generated in the duct. In the present study, the acoustic source was located upstream of the liner sample. Different nonintrusive measurement devices can then be added to access information on the acoustic fields inside the duct, such as microphones, or a transparent window through which laser measurements can be performed (see Sec. IV.A). Once the acoustic fields have been measured, they can be compared to the numerical fields obtained by solving the set of equations controlling the acoustic wave propagation in the duct, as detailed in Sec. II.B.

\section{B. Resolution of the Direct Problem}

Obtaining the numerical acoustic fields necessary for the impedance-eduction strategy requires solving the equations of acoustic propagation in the presence of a grazing flow. To do so, the 2-D time-harmonic LEEs are used. The underlying model is briefly summarized here for convenience, but all details can be found in [8].

The LEEs, written in nonconservative form with an $e^{j \omega t}$ time dependence and assuming homentropic flow, are given by

$$
\boldsymbol{L} \varphi=0
$$

in which

$$
\boldsymbol{L} \varphi=j \omega \varphi+\boldsymbol{A}_{x} \frac{\partial \varphi}{\partial x}+\boldsymbol{A}_{y} \frac{\partial \varphi}{\partial y}+\boldsymbol{B} \varphi
$$

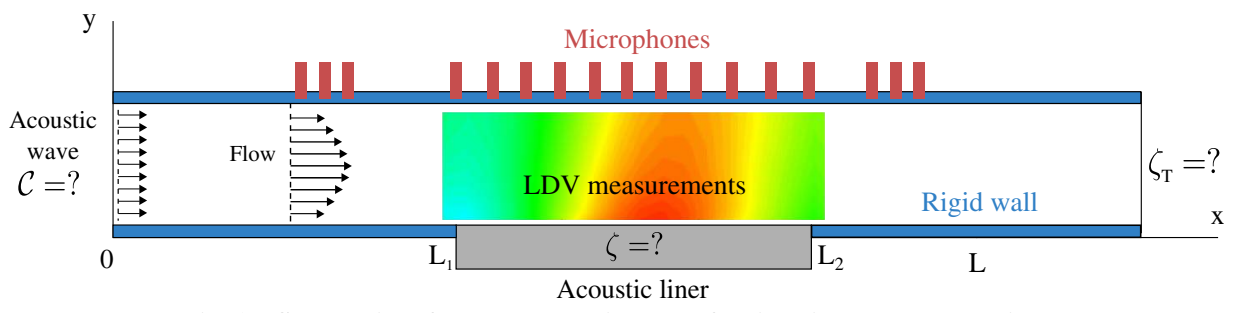

Fig. 1 Schematics of an aeroacoustic bench for liner impedance eduction. 
and

$$
\boldsymbol{A}_{x}=\left(\begin{array}{ccc}
U & 0 & c_{0} \\
0 & U & 0 \\
c_{0} & 0 & U
\end{array}\right), \boldsymbol{A}_{y}=\left(\begin{array}{ccc}
V & 0 & 0 \\
0 & V & c_{0} \\
0 & c_{0} & V
\end{array}\right), \quad \boldsymbol{B}=\left(\begin{array}{ccc}
\partial_{x} U & \partial_{y} U & 0 \\
\partial_{x} V & -\partial_{x} U & 0 \\
0 & 0 & 0
\end{array}\right)
$$

Components of vector $\varphi=\left(u, v,\left(p / \rho_{0} c_{0}\right)\right)^{T}$ represent the acoustic perturbations around the shear mean flow of axial and transverse velocities $U$ and $V$, respectively. Because of the homentropy condition, the energy equation is replaced by the state equation $p=c_{0}^{2} \rho$, with $\rho$ the acoustic density.

As we consider a shear mean flow profile, the impedance boundary condition on the lined wall is the standard one:

$$
p= \pm \rho_{0} c_{0} \zeta v
$$

in which the sign is chosen depending on whether the upper or lower wall is considered. To avoid any singularities in the rigid-wall case, the boundary condition (3) is expressed as a function of the normal incidence reflection coefficient $\beta=(\zeta-1) /(\zeta+1)$, leading to the following boundary-condition matrix:

$$
\boldsymbol{M}_{\beta}=\frac{1}{2}\left(\begin{array}{cc}
(\beta+1) \boldsymbol{n} \otimes \boldsymbol{n} & (\beta-1) \boldsymbol{n} \\
-(\beta+1) \boldsymbol{n}^{T} & (1-\beta)
\end{array}\right)
$$

in which $\boldsymbol{n}$ is the local normal vector, and $\otimes$ is the tensor product. On the rigid walls, the reflection coefficient $\beta$ is then taken as 1 .

At the source plane, the state vector $\varphi$ is taken to be equal to $\mathcal{C} \varphi_{0}$, in which $\varphi_{0}$ is the plane-wave solution in a rigid duct with uniform flow Mach number $M_{b}$, corresponding to the bulk Mach number of the actual shear flow $U(y)$ that was calculated by averaging the measurements performed at center span and three different $x$ locations. As this plane-wave solution is chosen with an arbitrary amplitude of 1 , the complex coefficient $\mathcal{C}$ determines the source amplitude and phase. $\mathcal{C}$ can be either set to the measured value of the incident acoustic pressure at the source plane, when available, or included into the eduction process. At the inlet boundary conditions, the two enforced boundary conditions required by the 2-D LEE are the specified pressure $p$ and $v=0$. At the exit plane, either a nonreflecting or an impedance boundary condition (of impedance $\zeta_{T}$ ) can be enforced. The first option is only used when the data suggest that this is proper (i.e., when no standing wave can be observed in the downstream hard-wall section). This information can only be provided by microphone measurements downstream of the lined section. Because these additional measurements are not systematically performed, the exit-impedance boundary condition is educed in all the computations shown in this paper. As a consequence, during the search procedure, both $\mathcal{C}$ and $\zeta_{T}$ are used as design variables, in addition to $\zeta$, thus totaling six variables.

A DG scheme is chosen to solve Eq. (1) and the associated boundary conditions. In this method, discontinuities are allowed at the interface between two elements. An example of how such a method can handle discontinuities at hard-soft wall interfaces is found elsewhere $[18,19]$. To ensure connection between elements and to apply the boundary conditions, a numerical flux is defined. An upwind numerical flux is used to ensure connection between interior cells, whereas a centered flux is chosen at the boundaries (see previous papers $[\underline{8}, \underline{12}]$ for more details).

\section{Bayesian Inference Applied to Liner Eduction}

Although most previous studies in impedance eduction focused on the minimization of a cost function $[6,8]$, representing the mismatch between numerical and experimental fields (pressure and velocity), little effort has been dedicated to the evaluation of uncertainties in the results $[14,15]$, associated with the measurement uncertainties inherent to the experimental observation. The method developed in the present study relies on a statistical approach to inverse problems, using Bayes's theorem. The parameters of interests are recast into random variables, and their posterior PDF becomes the quantity to be found. This technique offers the advantages of including experimental uncertainties into the search procedure and provides an elegant way to include prior knowledge in the modeling (such as measurements from another experiment or made by another laboratory, or common knowledge on material properties).

\section{Bayesian Approach to Inverse Problems}

Using Bayes's theorem, we define a posterior probability density $\pi\left(\boldsymbol{m} \mid \boldsymbol{d}^{\text {exp }}\right)$ for the model parameter $\boldsymbol{m}$, given a set of observable parameters $\boldsymbol{d}^{\exp } \in \Omega_{\mathrm{obs}}$. Formally, this reads

$$
\pi\left(\boldsymbol{m} \mid \boldsymbol{d}^{\exp }\right) \propto \overbrace{\pi\left(\boldsymbol{d}^{\exp } \mid \boldsymbol{m}\right)}^{\text {likelihood }} \overbrace{\pi(\boldsymbol{m})}^{\text {prior }}
$$

where $\boldsymbol{m}=\left(\operatorname{Re}(\zeta), \operatorname{Im}(\zeta),|\mathcal{C}|, \theta_{\mathcal{C}}, \operatorname{Re}\left(\zeta_{T}\right), \operatorname{Im}\left(\zeta_{T}\right)\right)$, in which $\zeta$ is the complex impedance of the liner, $|\mathcal{C}|$ and $\theta_{\mathcal{C}}$ are the amplitude and phase of the inlet plane wave, and $\zeta_{T}$ is the exit-impedance condition. Information on the physical correlations between $\boldsymbol{d}^{\exp }$ and $\boldsymbol{m}$ is predicted by a physical model represented by a deterministic forward observation operator $\boldsymbol{G}: \mathbb{R}^{n_{m}} \rightarrow \mathbb{R}^{n_{d}}$, in which $n_{m}=6$ is the number of parameters to infer, and in which $n_{d}$ is the number of measurement points, depending on whether microphone or LDV measurements are performed. A statistical model is added to account for the measurement noise and other sources of uncertainties. The forward operator has been described in Sec. II.B with a set of PDEs, solved with a DG scheme. The notation $\boldsymbol{G}$ is used to represent the whole process of calculating the solution of the LEE and extracting the numerical fields at the measurement points.

The experimental data considered in this study are either the pressure measurements on the wall opposite the liner, or the velocity fields above the liner found by LDV measurements. The measured data $d^{\exp }$ are related to the model parameters through

$$
\boldsymbol{d}^{\exp }=\boldsymbol{G}\left(\boldsymbol{m}^{*}\right)+\boldsymbol{\epsilon}
$$

with $\boldsymbol{m}^{*}$ unknown, in which $\boldsymbol{G}$ is the deterministic forward observation operator (dependent on whether pressure measurements or LDV measurements are used), and $\boldsymbol{\epsilon}$ accounts for measurement uncertainties, with components of $\boldsymbol{\epsilon}$ being independent and identically distributed random variables of density $\pi_{\epsilon}$ (also case dependent). For brevity of notation, we shall write the arrays $i$ th components, $d_{i}^{\exp }$ and $G_{i}(\boldsymbol{m})$. The likelihood can be written, assuming $\boldsymbol{\epsilon}$ follows a normal distribution, as

$$
L(\boldsymbol{m})=\prod_{i=1}^{n_{d}} \frac{1}{\sqrt{2 \pi \sigma_{i}^{2}}} \exp \left(-\frac{\left|d_{i}^{\exp }-G_{i}(\boldsymbol{m})\right|^{2}}{2 \sigma_{i}^{2}}\right)
$$

in which $\sigma_{i}^{2}$ denotes the noise variance, and is either determined experimentally or set up a priori. In the present study, $\sigma_{i}^{2}$ has been estimated for the pressure measurements by assuming a $\pm 0.5 \mathrm{~dB}$ uncertainty in measured amplitude and a \pm 1 deg uncertainty in measured phase, whereas for the LDV measurements, a bootstrap approach has been followed, as in Léon et al. [17], to provide an approximation of experimental variance for the velocity-field measurements (see Sec. IV.B). The prior probability $\pi(\boldsymbol{m})$ contains statistical information obtained on the model parameters independently of the experimental data used during the inference. It reflects the user's insight on the problem and known physical constraints. When no a priori information is available, a homogeneous probability density of large range represents well one's ignorance. In the present study, we assume prior independence between the unknown parameters, setting

$$
\pi(\boldsymbol{m})=\prod_{i=1}^{n_{m}} \pi_{i}\left(m_{i}\right)
$$

Prior information is available from previous measurements, but testing the present method in an objective way requires the 
Table 1 Prior bounds for a uniform probability

\begin{tabular}{lcccccc}
\hline \hline Parameter & $\operatorname{Re}(\beta)$ & $\operatorname{Im}(\beta)$ & $|\mathcal{C}|$ & $\theta_{\mathcal{C}}$ & $\operatorname{Re}\left(\beta_{T}\right)$ & $\operatorname{Im}\left(\beta_{T}\right)$ \\
\hline$m_{i, \min }$ & -1 & -1 & 0.1 & $-\pi$ & -1 & -1 \\
$m_{i, \max }$ & 1 & 1 & 2.0 & $+\pi$ & 1 & 1 \\
\hline \hline
\end{tabular}

use of a noninformative uniform prior probability $\left(\pi_{i}\left(m_{i}\right) \sim\right.$ $\left.\mathcal{U}\left(m_{i, \min }, m_{i, \max }\right)\right)$, in which the lower and upper bounds are given in Table 1. This type of statistical inference is called objective firstlevel B̄ayesian inference [20], because we apply Bayesian data analysis to solving a parameter-calibration problem, neglecting the second inference level of selecting a model simultaneously. Using a broad or a flat prior reflects maximal prior uncertainty, or noninformative prior knowledge, in which only the newly observed data drive the Bayesian analysis and inform the posterior densities, through the likelihood function. The posterior PDF is now rewritten in its full form as

$$
\pi\left(\boldsymbol{m} \mid \boldsymbol{d}^{\exp }\right)=\frac{1}{\Xi} \pi(\boldsymbol{m}) L(\boldsymbol{m})
$$

in which $\Xi=\pi\left(\boldsymbol{d}^{\exp }\right)=\int_{m} \pi\left(\boldsymbol{d}^{\exp } \mid \boldsymbol{m}\right) \pi(\boldsymbol{m})$ is a normalizing factor, also called Bayesian evidence, which represents the probability that the data $\boldsymbol{d}^{\exp }$ be generated by this model. The precise integration of this term is problematic, especially when the dimension of $\boldsymbol{m}$ is high. To palliate this issue, an MCMC technique is used to draw samples from an unnormalized posterior probability.

\section{Markov Chain Monte Carlo}

The central idea behind the MCMC methods is to draw samples directly from the posterior distribution without having to calculate the normalizing factor. A random-walk algorithm is designed to explore the posterior density by moving preferentially to regions of high probability densities. A Metropolis-Hastings algorithm [21,22] is used to create a Markov chain whose stationary distribution is precisely the distribution of interest [23].

The MCMC strategy is coupled with a differential-evolution algorithm [24]. Multiple Markov chains are run in parallel and learn from each other through mutation and swap operators, thus solving the problems of selecting a direction and a scale for the jump from the current point to the proposal point [25]. In addition, a multitry approach, a sampling from past chain states, a self-adaptive randomized subspace sampling, and a snooker update have been integrated in our procedure, as detailed in [26,27], which allows the reduction of the number of chains, from $2 n_{m}$ down to $N_{\text {chains }}=3$. To drastically accelerate the sampling, a surrogate model is first defined over the full scope of the prior, as detailed in Sec. II.C.3.

In the following impedance eductions, $10^{5}$ iterations are performed for the three chains, and the first $10 \%$ samples of each chain posterior are discarded as burn-in [23] to let the Markov chain forget its initial state and converge to a unique stationary distribution. The chains are then merged, resulting in the posterior matrix containing $n_{m}$ columns and $N_{\text {eval }}=N_{\text {chains }} \times(1-0.1) \cdot 10^{5}$ rows. From this, histograms of the parameter values can be extracted to approximate the true target densities of interest.

\section{Surrogate Modeling}

In the present case, following a statistical inference approach would require $N_{\text {eval }}$ evaluations of the system of PDEs described by Eq. (1). Doing so would be prohibitively long, especially because we wish to look at a large number of frequencies and different Mach numbers. Without any acceleration, a single evaluation of the observation operator on the PDEs takes about a second. Although it may seem fast, doing $N_{\text {eval }}$ function evaluations would still require about $10 \mathrm{~h}$ with our current eight-processor machine, for each frequency/Mach number. (The developed MCMC strategy can be fully parallelized.) To palliate this shortcoming, an efficient representation of the discretized state of the system $\varphi$ is obtained at a lower cost by first building a reduced basis using the method of snapshots, as explained by Pinnau [28] and used for Bayesian inference by Cui et al. [29]. The approach is summarized again here for completeness. The full discretized DG system reads:

$$
j \omega \varphi+A_{x} \partial_{x} \varphi+A_{y} \partial_{y} \varphi=0
$$

in which $A_{x}, A_{y}$ are of size $\mathbb{R}^{N \times N}$, with $N$ the system dimension, depending on the number of field variables (here, three), the number of mesh elements (here, $~ 8000$ ), and the DG order (here, $p=2$ ). The aforementioned discretization is parameterized by the unknown parameters looked for in the eduction process. A set of $n$ parameter samples $\left\{\boldsymbol{m}^{(1)}, \ldots, \boldsymbol{m}^{(n)}\right\}$ is generated, yielding the associated system states, or snapshots: $\left\{\varphi^{(1)}, \ldots, \varphi^{(n)}\right\}$. A reduced basis $V_{m}=$ $\operatorname{span}\left(\varphi^{(1)}, \ldots, \varphi^{(n)}\right)$ is then created. Approximating Eq. (10) by a Galerkin projection yields

$$
j \omega \varphi_{m}+V_{m}^{t} A_{x} \partial_{x} V_{m} \varphi_{m}+V_{m}^{t} A_{y} \partial_{y} V_{m} \varphi_{m}=0
$$

This allows the approximation of the state $\varphi$ by a linear combination of vector elements of $V_{m}$ as $\varphi \approx V_{m} \varphi_{m}$. When the number of snapshots $n$ is low, then $\operatorname{dim}\left(V_{m}\right) \ll N$, and the reduced system represented by Eq. (11) is much faster to solve than the full system. Unlike Cui et al. [29], the present surrogate approach is based on the prior bounds. Our different convergence analyses (not shown therein for conciseness) showed that as little as 30 randomly selected samples in the parameter space were large enough to create an accurate reduced basis over the prior, due to an exponential decrease in the eigenvalues of the correlation matrix associated with the snapshots [28]. A speed-up factor of about $10^{3}$ is thus obtained. Some additional bias is bound to be present in the surrogate Monte Carlo estimator due to the approximate distribution that is now sampled from. However, a little bias is acceptable when the speedup is enough so as to allow an overall reduction in the mean square error of the posterior expectation, for a given numerical budget [29].

\section{Eduction Strategy Validation on NASA Grazing Incidence Tube Benchmark}

\section{A. Generalities}

NASA provided a quality data set that can be used as a benchmark for evaluation of duct propagation and impedance-eduction codes [6]. In this section, the developed Bayesian inference method is compared with the least-mean-squares (LMS) deterministic approach used by NASA [6]. In addition to the difference in eduction method considered, the strategies differ in two major ways. First, the present study considers a shear grazing flow, thus solving the LEE, instead of considering a uniform grazing flow as with the CHE. Furthermore, the identified parameters consist in four additional variables, because the present work educes the termination impedance and the source simultaneously, whereas, previously [6], they were measured in situ. Although the source needs to be educed, because its value is unknown, a different approach could have been taken regarding the inference of the termination impedance, since it has been measured. However, educing this extra parameter in a controlled eduction problem allows for further validation of our inference strategy.

The liner consists in a porous material made of a ceramic tubular matrix of porosity $57 \%$, coined CT57. This material is known for having a linear behaviour relative to both the sound-pressure level (SPL) and the Mach number. It presents the advantage of displaying a wide range of impedance values, which is interesting when testing a new inference method for impedance eduction.

\section{B. Eduction Results}

Eduction results obtained at NASA using a deterministic LMS approach are compared with the ones obtained in this work using the Bayesian inference technique consist here of a set of 31 pressure measurements placed on the wall opposite the liner. Although the exit-impedance condition has been measured directly by NASA and is close to a nonreflection condition, it is still assumed unknown here, 
to validate the educed values. However, to further regularize the inverse problem, a prior probability is assigned to the value of the exit impedance. The mean and variance of the direct experimental measurements of the exit impedance are used to assign a Gaussian prior probability to the exit impedance. The source amplitude and phase remain unknown, and must therefore enter the identification process as well, even though their identification is not of particular interest for the present study. (They are coined nuisance parameters in the literature [30].) The shear grazing-flow profile is fitted to the experimentally measured one using an analytical profile of Štigler [31]. The analytical profile is scaled so that the bulk Mach number is the one used in Ref. [6].

At every frequency, and for each Mach number, the Bayesian inference is performed. The output of such a method is a matrix containing all the samples obtained by the MCMC technique detailed in Sec. II.C.2. From these samples, one can calculate different statistical moments, such as the mean or variance, or credibility intervals (CIs) for the inferred parameters. In the context of Bayesian inference, a $95 \% \mathrm{CI}$ is interpreted as a $95 \%$ probability that the true value of a given parameter falls within the credible region.

Impedance-eduction results at two different Mach numbers are given in Fig. 2, in which the $95 \%$ intervals obtained by the Bayesian inference are compared against the NASA deterministic eduction approach (symbols). The eduction is performed on the reflection coefficients, and the impedance is calculated as a post-treatment using the relationship, $\zeta=(1+\beta) /(1-\beta)$. Both the reflection coefficient $\beta$ and the impedance $\zeta$ real and imaginary parts are displayed. For the inference procedure, using the reflection coefficient instead of the impedance allows the reduction of the parameter space size, because $\beta$ is bounded, whereas $\zeta$ is not. For both Mach numbers considered $\left(M_{b}=0\right.$ and $\left.M_{b}=0.255\right)$, the bars corresponding to the $95 \%$ CIs are not easily visible on the graph, except around the antiresonance of the system. This is explained in the following way: at the antiresonance, the resistance (real part of the impedance) is high and the reactance (imaginary part of the impedance) becomes 0 , with a negative reactance slope. The liner is seen by the acoustics as an almost rigid wall. As such, the likelihood function is less sensitive to impedance variations at high values. As a general rule, the uncertainties at $M_{b}=0.255$ are greater than when no flow is present, which is easily explained by the added noise in the measurements. However, the general trend of increased uncertainty at antiresonance is also present. At the antiresonance and at $M_{b}=0.255$, the value of the real part of the impedance, educed using Bayesian inference, is lower than the reference value. This is possibly due to the difference in flow profile (grazing vs uniform).

The inferred values for the termination impedance $\zeta_{T}$ are displayed in Fig. 3 , in which a perfect agreement between measured and educed values is obtained for the case $M_{b}=0$. However, the inverse measurement of $\zeta_{T}$ at $M_{b}=0.255$ appears much more inaccurate than what has been obtained for the liner impedance. This is in part due to the exit termination mostly only affecting the last three microphones, downstream the liner, where a standing wave pattern can be created. Different tests (not shown for succinctness) were performed to study the influence of changing the prior probability imposed on the termination impedance. Although the termination impedance itself was sensitive to this change, the liner impedance remained practically unchanged. Overall, the agreement between eduction methods tends to show that the present statistical inference technique provides fairly accurate and informative results for the liner impedance. Although these results take the form of a PDF, only the 95\% CIs were represented in Fig. 2. The close examination of Fig. 2 shows that, despite the use of a linear porous liner, a deviation in both the real and imaginary parts of the impedance is seen when the flow velocity increases: an increase in Mach numbers tends to shift the
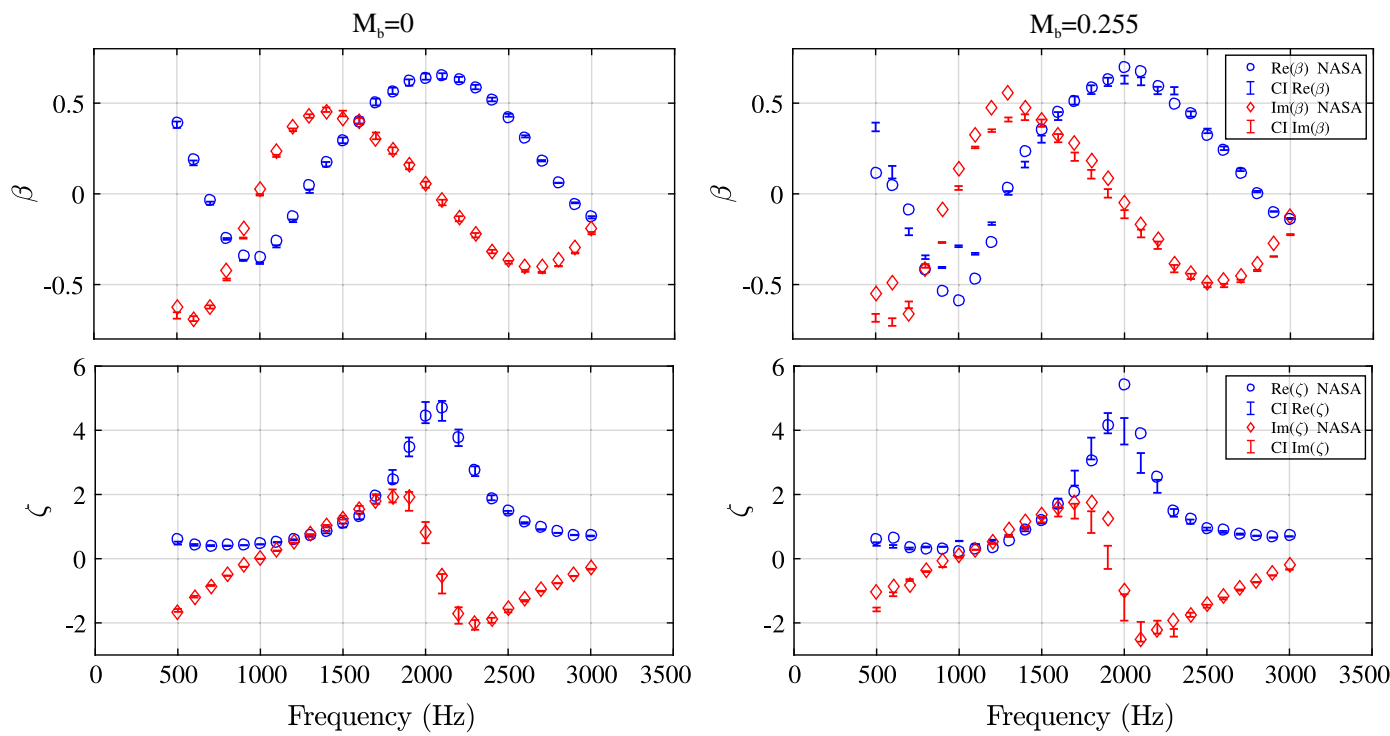

Fig. 2 Comparison between NASA eduction method and Bayesian inference results.
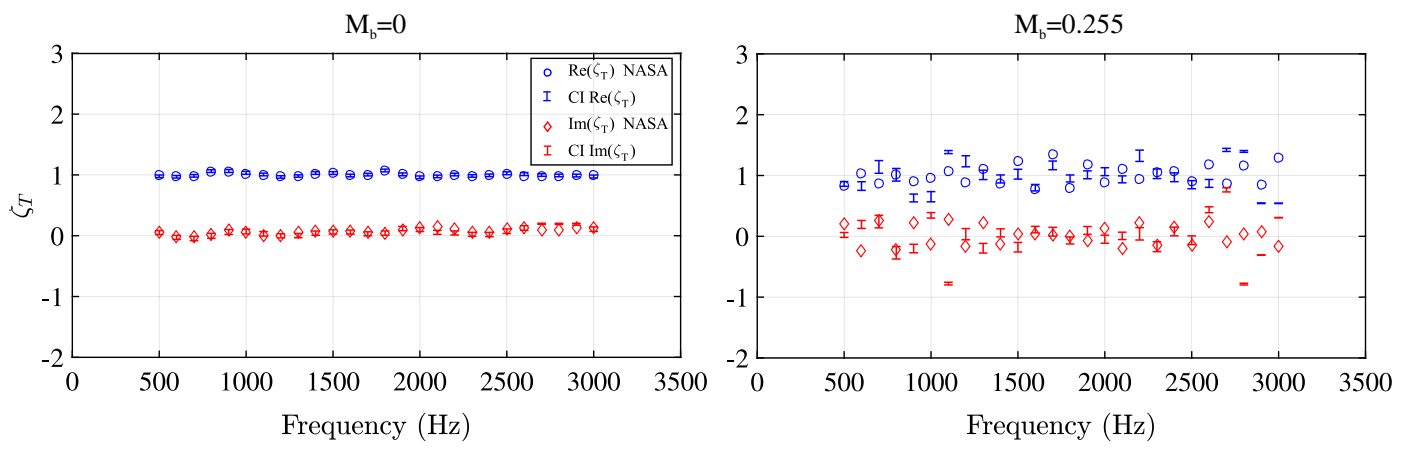

Fig. 3 Comparison between deterministic and Bayesian inference results for $\zeta_{T}$. 
resistance and reactance to lower frequencies. This has been adressed in a previous study by Jing et al. [32], who showed that this was due to the velocity profile definition in the $2-\mathrm{D}$ case.

\section{Analysis at the Resonance}

The liner resonance (i.e., when the reactance becomes 0 and the reactance slope is positive, around $1000 \mathrm{~Hz}$ ) is of particular interest, because this is where the acoustical attenuation is usually close to the maximum in the absence of flow. When a shear grazing flow is present in the duct, this is not the case anymore, but we consider the resonance to be a reference point, useful when making comparisons later on. Thus, a more detailed analysis is conducted at $f=1000 \mathrm{~Hz}$, for a Mach number of $M_{b}=0.255$, on the NASA CT57 sample. Results are displayed in the form of the posterior PDF and joint PDFs between parameters, as shown in Fig. 4. Diagonal elements are obtained by applying a kernel density estimator on the histograms resulting from the MCMC posterior sampling. The 2-D joint PDF (off-diagonal elements) were calculated by first binning the scatterplot of each parameter pair, calculating the associated 3-D histogram, and then applying a surface plot to display the binned areas of high probability densities, with warmer colors corresponding to higher probability densities. The goal of such a plot is to see the shape of the densities and the density support range of the sampled MCMC elements. This can also be used to describe the correlations that exist between the parameters for a specific inverse problem. From these results, it can be seen that there exists, for this set of measurements and likelihood function, a strong correlation between $\operatorname{Re}(\zeta)$ and $|\mathcal{C}|$ on the one hand, and $\operatorname{Im}(\zeta)$ and $\theta_{\mathcal{C}}$ on the other hand. (A thin elliptic diagonal joint PDF shape indicates a strong correlation between parameters.)

Those particular correlations can be interpreted as follows. Most of the observation is realized on the wall directly opposite the liner, giving a weight, or contribution, to the likelihood, which is more important than the few measurement points upstream and downstream. (On the 31 microphones used, 25 are directly above the liner.) As a result, modulating the amplitude and phase of the source affects the likelihood function on the measurement positions directly opposite the liner, where the influence of the impedance is incidentally also the strongest.

These four competing variables can thus share a similar influence on the field at the wall opposed to the liner region: lowering the source amplitude lowers the whole pressure field amplitude in the aeroacoustic bench, but is mostly only observed upstream the liner, where changing the resistance can have a similar effect. Similarly, changing the source phase or the liner reactance (interpreted as a delay, or time shift in time domain) has comparable effects on the observation. The appearance of strong correlations between these four parameters has been confirmed at all frequencies, but is not shown here for conciseness.

This general result shows that having a somewhat biased value for the source parameters would likely result in a biased estimation of the impedance. As such, it is recommended to perform some prior measurements on the source, in the same way the exit-impedance condition can be measured before the eduction process. We note that the termination impedance does not appear to be strongly correlated with any other parameter, but that its real and imaginary parts are correlated. This correlation seems to be caused by the addition of a prior law to the termination impedance, as it was absent in the tests we performed without prior.

\section{Application to an SDOF Liner with LDV}

\section{A. Experimental Setup}

\section{Aeroacoustic Bench: B2A}

The ONERA B2A flow duct is a 4-m-long stainless-steel tube of square section $50 \times 50 \mathrm{~mm}$, where the 0.2 -m-long test section is equipped with silica windows for optical access. The termination is equipped with a quasi-anechoic outlet, leading to an upstream

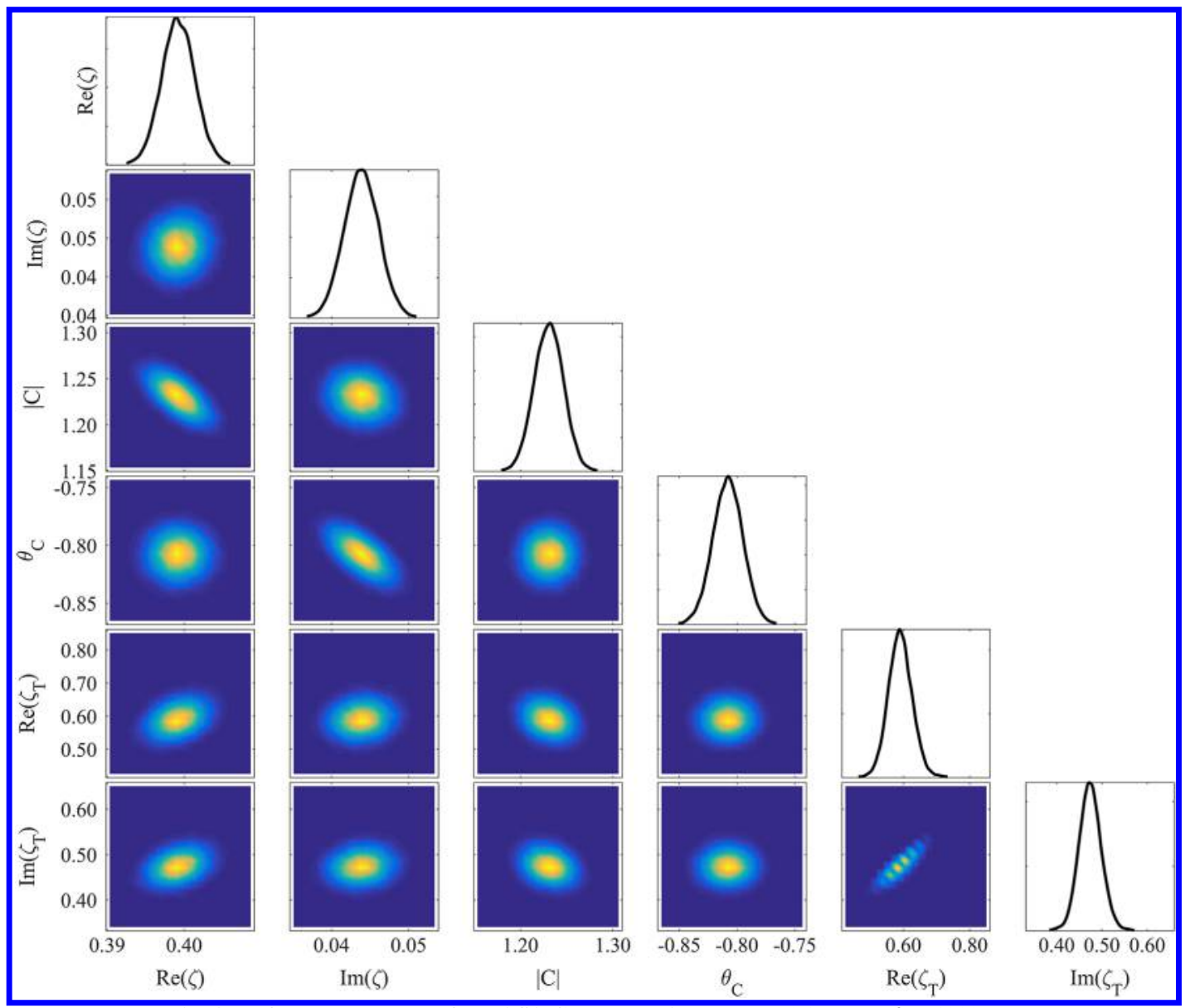

Fig. 4 Inferred parameters for NASA CT57 material at $M_{b}=0.255$ and $f=1000 \mathrm{~Hz}$. 


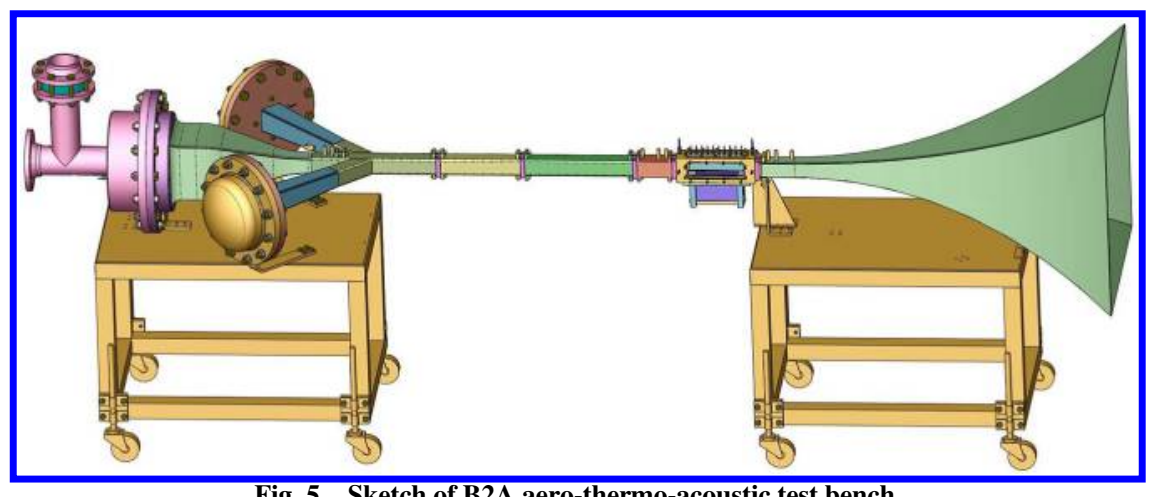

Fig. 5 Sketch of B2A aero-thermo-acoustic test bench.

reflection coefficient smaller than 0.2 for frequencies higher than $500 \mathrm{~Hz}$. A fully developed turbulent duct flow of bulk Mach numbers up to 0.6 can be provided, where the turbulence rate is of a few percent at the center of the test section. A schematic of the B2A flow duct is provided in Fig. 5 , in which the flow propagates from left to right. Two acoustic drivers are mounted upstream of the test section to generate multisine tones at up to $150 \mathrm{~dB}$ over a frequency range of $0.3-3.5 \mathrm{kHz}$. The surface of the test liner forms a $150-\mathrm{mm}-\mathrm{long}$ portion of the duct lower wall. Six microphone locations and two static pressure taps are available in the upper wall to measure the pressure field upstream of the liner leading edge and downstream of the liner trailing edge. The upper silica window (facing the liner) can be replaced by a hard wall equipped either with 10 additional microphone locations, or with 10 static pressure taps. Flushmounted Brüel \& Kjær (B\&K) 4182 microphonic probes are used (probe diameter of $1.2 \mathrm{~mm}$ ). The upstream and downstream microphone locations can be used to measure the amplitude of the incident and transmitted acoustic plane waves [33]. The cross spectrum between the microphones and the driver signal is acquired, which provides a phase reference for the acousticpressure measurements.

\section{LDV for Impedance Eduction}

A two-component fringe-mode LDV allows the measurement of the axial and vertical velocity components in almost the entire volume of the test section [34]. The emitting optics produce an elliptical measurement volume whose little axis can be as small as $50 \mu \mathrm{m}$. Flow is seeded with either incense smoke or amorphous silica particles. The LDV signal is unevenly sampled due to the random arrival of particles in the measurement volume. A reconstruction method [35] is used to resample the raw data at a constant rate. Signals are processed using the in-house ONERA software,
ASSA [36]. A minimum sampling rate of $f_{m}=13,000$ measurements per second is ensured, for each velocity component, and more than 200,000 samples are acquired.

The present experiments were performed under normal atmospheric conditions, with the flow temperature regulated at the ambient temperature $T_{0}=20^{\circ} \mathrm{C}$ with maximum variations of $1^{\circ} \mathrm{C}$, yielding a speed of sound of $c_{0}=343 \mathrm{~m} / \mathrm{s}$. The mass-flow rate was set to $Q=260 \mathrm{~g} / \mathrm{s}$, corresponding to a bulk velocity $U_{b}=$ $89.18 \mathrm{~m} / \mathrm{s}$ and a bulk Mach number $M_{b}=0.26$. The acoustic signal is a multisine signal composed of 12 frequencies (in $\mathrm{Hz}$ ): 504, 616, 760, 992, 1112, 1240, 1488, 1736, 1992, 2248, 2488, and 2824.

For each frequency value, a wave sorting procedure is applied to obtain an incident SPL of $130 \mathrm{~dB}$ on each tone. It consists of applying a two-microphone method [33] to decompose the sound field in the duct into an incident and a reflected wave. Two microphone probes B\&K 4182 are placed flush to the flow, at a distance from the sample. The spacing between the two probes is $2.8 \mathrm{~cm}$, which, according to Boden and Abom [37], minimizes errors in the [600, 3450] Hz frequency range. To also minimize measurement errors at lower frequencies, the measurements are repeated by switching the two microphones, and an average of the obtained frequency response function is taken: this makes it possible to overcome the intrinsic phase shift between the microphones. The LDV measurement data set represents more than 325 LDV data points. The LDV system enables to measure 2-D velocity fields. The measurement plane is positioned at $z=0$, which corresponds to the center of the test section in the spanwise direction. Only $(\tilde{U}, \tilde{V})$ are measured in the $(x, y)$ coordinate system. An example of the norm of the acoustic velocity is shown in Fig. 6 . The liner is $150 \mathrm{~mm}$ long, and is located between $x=0$ and $x=150 \mathrm{~mm}$, at $y=0 \mathrm{~mm}$. Estimations of confidence intervals on the LDV measurement can be obtained with a bootstrap technique (see Sec. IV.B).

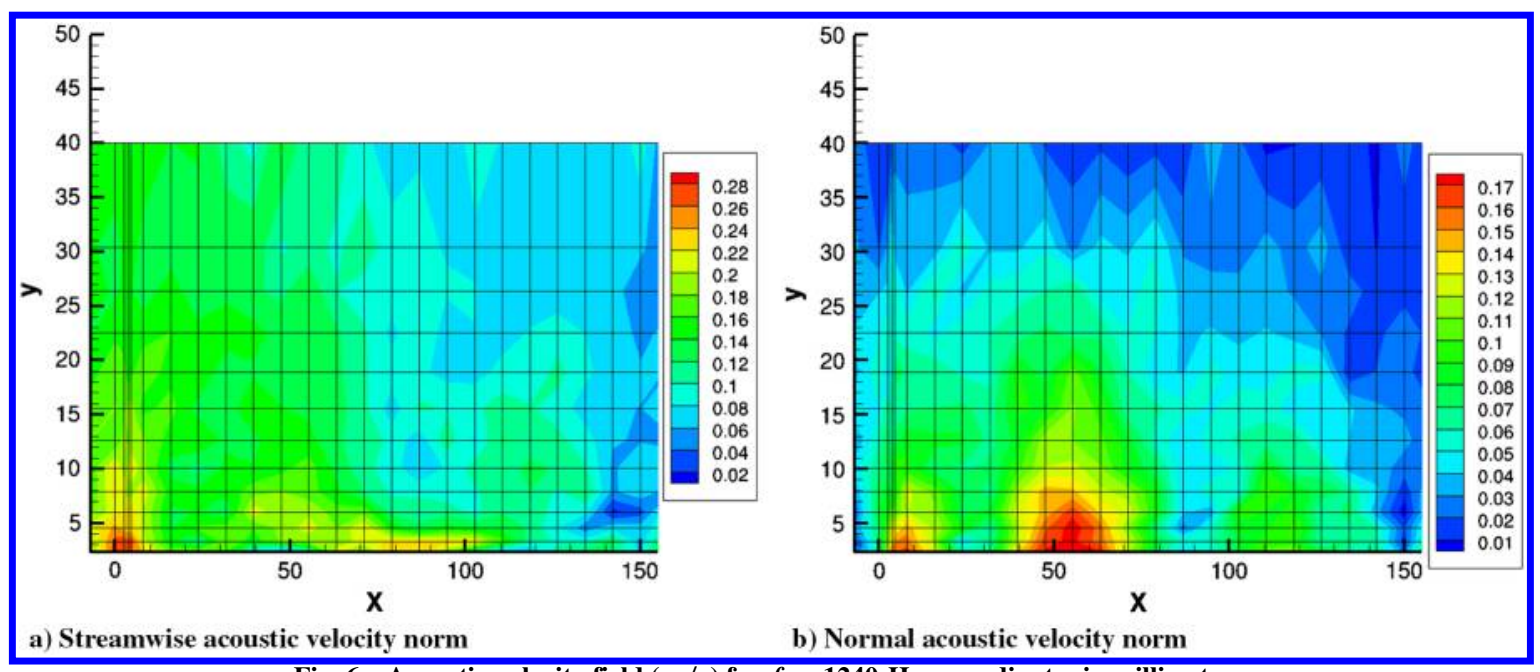

Fig. 6 Acoustic-velocity field $(\mathrm{m} / \mathrm{s})$ for $f=1240 \mathrm{~Hz}$; coordinates in millimeters. 
3. Velocity Signal Postprocessing

The velocity fields $(\tilde{U}, \tilde{V})$ can be decomposed into four parts, as

$$
\begin{aligned}
& \left(\begin{array}{c}
\tilde{U} \\
\tilde{V}
\end{array}\right)(x, y, t)=\left(\begin{array}{l}
U \\
V
\end{array}\right)(x, y)+\left(\begin{array}{l}
u \\
v
\end{array}\right)(x, y, t)+\left(\begin{array}{l}
u^{\prime} \\
v^{\prime}
\end{array}\right)(x, y, t) \\
& +\left(\begin{array}{c}
b_{u} \\
b_{v}
\end{array}\right)(x, y, t)
\end{aligned}
$$

The turbulent velocity fluctuations are assumed uncorrelated with the acoustic fluctuations. The acoustic velocity is defined here as the signal component that is correlated with the loudspeaker signal. It can be separated from the extraneous noise (due to both the turbulent flow and the measurement noise) by a technique similar to the threemicrophone signal enhancement technique $[33,38]$. It consists in calculating the cross-spectral density function $\bar{G}_{\tilde{U}}, l s$ (respectively, $G_{\tilde{V}, l s}$ ) between the velocity signal $\tilde{U}$ (respectively, $\tilde{V}$ ) and the loudspeaker signal $l s(t)$ (see $[35,39,40]$ ). The autospectral density function of the acoustic velocity reads

$$
\begin{aligned}
G_{\tilde{U}} & =\frac{\left|G_{\tilde{U}, l s}\right|^{2}}{G_{l s}} \\
G_{\tilde{V}} & =\frac{\left|G_{\tilde{V}, l s}\right|^{2}}{G_{l s}}
\end{aligned}
$$

in which $G_{l s}$ is the autospectral density function of the loudspeaker signal. The acoustic velocities in the frequency domain are then given by

$$
\begin{aligned}
& u=\sqrt{G_{\tilde{U}}} \exp [j \Phi(u / l s)] \\
& v=\sqrt{G_{\tilde{V}}} \exp [j \Phi(v / l s)]
\end{aligned}
$$

in which the phase of the acoustic velocities, referenced by the loudspeaker, is defined as

$$
\begin{aligned}
& \Phi(u / l s)=\arctan \frac{\operatorname{Im}\left(G_{\tilde{U}, l s}\right)}{\operatorname{Re}\left(G_{\tilde{U}, l s}\right)} \\
& \Phi(v / l s)=\arctan \frac{\operatorname{Im}\left(G_{\tilde{V}, l s}\right)}{\operatorname{Re}\left(G_{\tilde{V}, l s}\right)}
\end{aligned}
$$

The approach retained in the present work to evaluate an estimator $\widehat{G_{\tilde{U}, l s}}\left(\right.$ respectively, $\widehat{G_{\tilde{V}, l s}}$ ) of the cross-power spectral function $G_{\tilde{U}, l s}$ (respectively, $G_{\tilde{V}, l s}$ ) relies on a Welch's overlapped segmented average (WOSA) method. For the acoustic-velocity amplitude estimator $\widehat{G_{\tilde{U}, l s}}$ (respectively, $\widehat{G_{\tilde{V}}, l s}$ ), spectral leakage and amplitude errors yielded by the choice of a windowing function have to be taken into account for an accurate, unbiased estimation. The present approach uses the cross-power spectra with the appropriate effective noise bandwidth, which depends on the window function employed. To minimize the amplitude error, a flattop window is used. The evaluation of $\widehat{G_{\tilde{U}, l s}}$ (respectively, $\widehat{G_{\tilde{V}, l s}}$ ) is then directly performed at the closest frequencies of interest. Regarding the acoustic phasedifference estimator $\Phi(u / l s)$ (respectively, $\Phi(v / l s)$ ) the simplest method is to evaluate the phase of $\widehat{G_{\tilde{U}}, l s}$ (respectively, $\widehat{G_{\tilde{V}}, l s}$ ) at the closest desired frequency, on a single frequency bin. An exhaustive analysis of estimators is available in Léon et al. [17].

\section{SDOF Liner}

The liner sample used in this work is a conventional SDOF liner made of a resistive perforated sheet of percent open area (POA) $15 \%$, mounted onto square cavities. It has been manufactured through an additive metallic manufacturing process, at the dimensions of the $\mathrm{B} 2 \mathrm{~A}$ test cell $(50 \mathrm{~mm}$ width and $150 \mathrm{~mm}$ length). The samples are
Table 2 General geometric characteristics of the SDOF sample

\begin{tabular}{lc}
\hline \hline Name & Value \\
\hline$L_{\text {liner }}$ & $150 \mathrm{~mm}$ \\
$W$ & $50 \mathrm{~mm}$ \\
$H$ & $48 \mathrm{~mm}$ \\
$\delta$ & $1.5 \mathrm{~mm}$ \\
$t_{\mathrm{pp}}$ & $1 \mathrm{~mm}$ \\
POA & $15 \%$ \\
\hline \hline
\end{tabular}

made of EOS MaragingSteel MS1, which is a pre-alloyed ultrahighstrength steel in fine powder form. The geometric characterization of the liner sample is available in Table 2 . The different symbols are described in the Nomenclature.

\section{B. Uncertainty Quantification of the LDV Measurements}

The uncertainty level on the acoustic-velocity-field measurements is a major input of the Bayesian inference process. Léon et al. [17] showed the possibility to use a database method to estimate the confidence interval of LDV measurements, using a bootstrap method [41], to estimate the uncertainty level on the acoustic-velocity amplitude and phase. Bootstrap methods provide a way to evaluate the variance associated with some sample estimates through random sampling with replacement. In our case, because the estimation of the cross-power spectral function relies on a WOSA method, the sample that has to be considered is the data set made of all the spectra involved in the periodogram method. The estimates at play are obtained by averaging these spectra and taking the magnitude and phase of the result at the frequency of interest. We performed such an analysis using the bias-corrected accelerated (BCa) nonparametric method developed by Efron and Tibshirani [41]. We considered $10^{4}$ bootstrap samples, and evaluated the variance of both the acousticvelocity amplitude and phase-difference estimates obtained with LDV data. Regarding the data acquisition configuration, one can consider that this case is composed of normally distributed estimates. The confidence intervals then defined by $\pm 1.96 \sigma_{|u|}$ (respectively, $\pm 1.96 \sigma_{|v|}$ ) and $\pm 1.96 \sigma_{\Phi_{u}}$ (respectively, $\pm 1.96 \sigma_{\Phi_{v}}$ ) provide a $95 \%$ probability to contain the waveform parameter values. Figures $7 \mathrm{a}$ and $7 \mathrm{~b}$ illustrate the acoustic-velocity-field measurements and associated confidence intervals obtained with the bootstrap technique for two different frequencies $(504$ and $1736 \mathrm{~Hz})$ at a constant $y=$ $12 \mathrm{~mm}$ position above the liner. This position is only chosen for illustrative purposes. For the inference, the variance is calculated at all $(x, y)$ locations using the BCa technique. For a frequency where the liner behaves almost like a rigid wall (for instance, $f=504 \mathrm{~Hz}$ ), the normal acoustic-velocity amplitude is very low and the streamwise acoustic-velocity amplitude is slightly varying along the liner. Because the normal acoustic-velocity norm is low, the uncertainty is comparatively greater. A decrease of the acoustic velocity along the liner streamwise direction is evidenced near the resonant frequency $(1736 \mathrm{~Hz})$. An increase of the normal acousticvelocity amplitude is also evidenced, as the liner is reacting to the acoustic excitation. The uncertainty on the normal acoustic-velocity amplitude is thus decreased because the signal-to-noise ratio is better. The variances of the streamwise and normal acoustic velocities are then averaged on the full LDV mesh, and the results are displayed as a function of frequency in Fig. $\underline{8}$. Figure $\underline{8}$ exhibits clearly the frequency range where the liner is absorbing: the error on the streamwise acoustic-velocity amplitude is maximum because the velocity is decreased, and the error on the normal acoustic-velocity amplitude is minimum because the liner is absorbing and the normal velocity is thus increased.

Looking at Fig. $\underline{8}$ shows that the likelihood function of Eq. (7) must separate the contributions of the streamwise and normal velocity components, because they share different levels of uncertainty as a function of frequency. This is done by simply considering a composite likelihood function as 


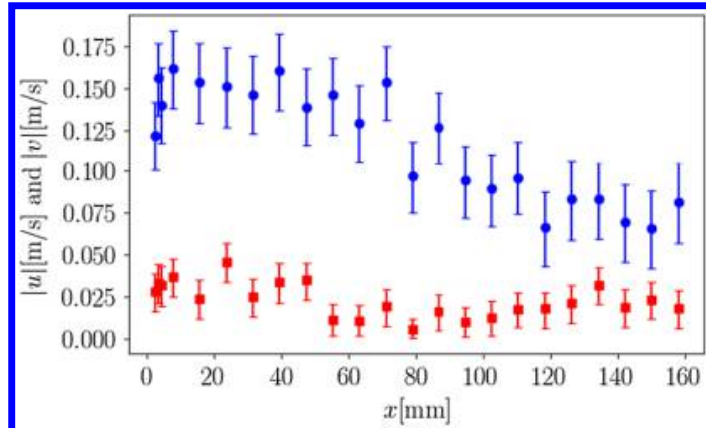

a) $|u|$ (blue circle), $|v|$ (red square), $f=504 \mathrm{~Hz}$

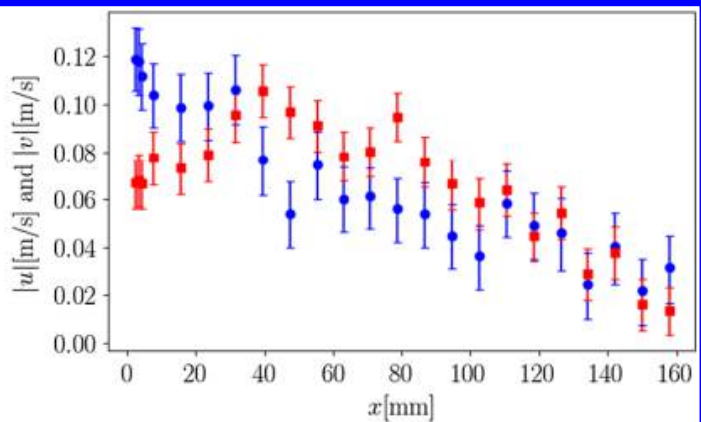

b) $|u|$ (blue circle), $|v|$ (red square), $f=1736 \mathrm{~Hz}$

Fig. 7 Acoustic-velocity with a 95\% confidence interval at $y=12 \mathrm{~mm}$.

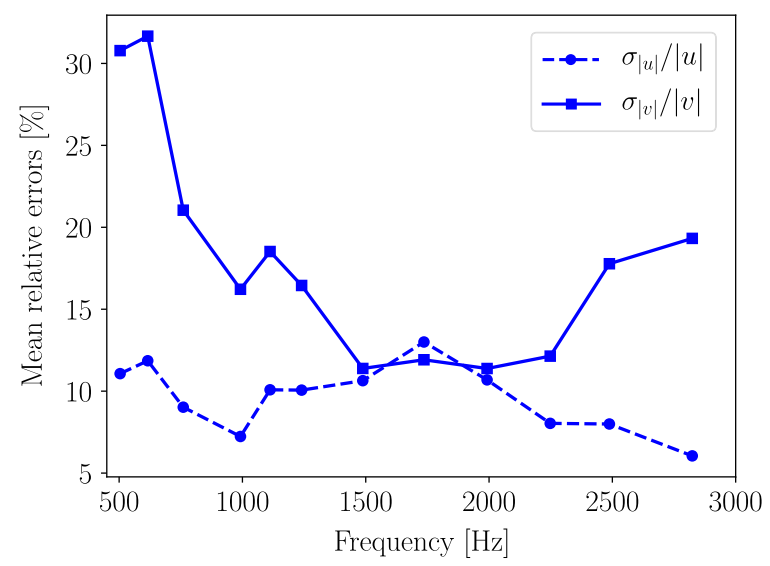

Fig. 8 Streamwise and normal acoustic-velocity variance value averaged along all measurement points.

$$
\begin{gathered}
L(\boldsymbol{m})=L_{u}(\boldsymbol{m}) \cdot L_{v}(\boldsymbol{m})=\prod_{i=1}^{n_{d}} \frac{1}{\sqrt{2 \pi \sigma_{u, i}^{2}}} \exp \left(-\frac{\left|u_{i}^{\text {exp }}-u_{i}^{\text {num }}\right|^{2}}{2 \sigma_{u, i}^{2}}\right) \\
\cdot \prod_{i=1}^{n_{d}} \frac{1}{\sqrt{2 \pi \sigma_{v, i}^{2}}} \exp \left(-\frac{\left|v_{i}^{\text {exp }}-v_{i}^{\text {num }}\right|^{2}}{2 \sigma_{v, i}^{2}}\right)
\end{gathered}
$$

in which the dependencies in the frequency and in $\boldsymbol{m}$ have been omitted for clarity. In practice, the log-likelihood is considered.

\section{Eduction Results}

Figure 9 presents the liner impedance-eduction comparison between the classical deterministic LMS approach (see Primus et al. [8] for instance) and the present Bayesian inference method taking into account the confidence intervals of the LDV measurements obtained by the bootstrap method (Sec. IV.B). An excellent agreement between both methods is obtained, when comparing the mean of the inference results to the optimum of the deterministic approach. Because the posterior PDF were all Gaussian in this case, the mean is located at the center of the CIs in Fig. 9. The statistical inference approach shows a larger CI on the educed impedance at low frequencies, which is a good indicator of the inversion being more challenging in this frequency range. This is in part due to the streamwise extension of the LDV measurement window, and in part because of the low absorption resulting from the liner at these frequencies. It is believed that combining microphone measurements to LDV measurements could yield an improved eduction strategy, provided that the spacing between upstream and downstream microphones (compared to the lined section) is large enough to capture the variations of low-frequency waves. Alternatively, one could consider the present posterior PDF, obtained by LDV-based inference, as a prior probability for a next inference, which would

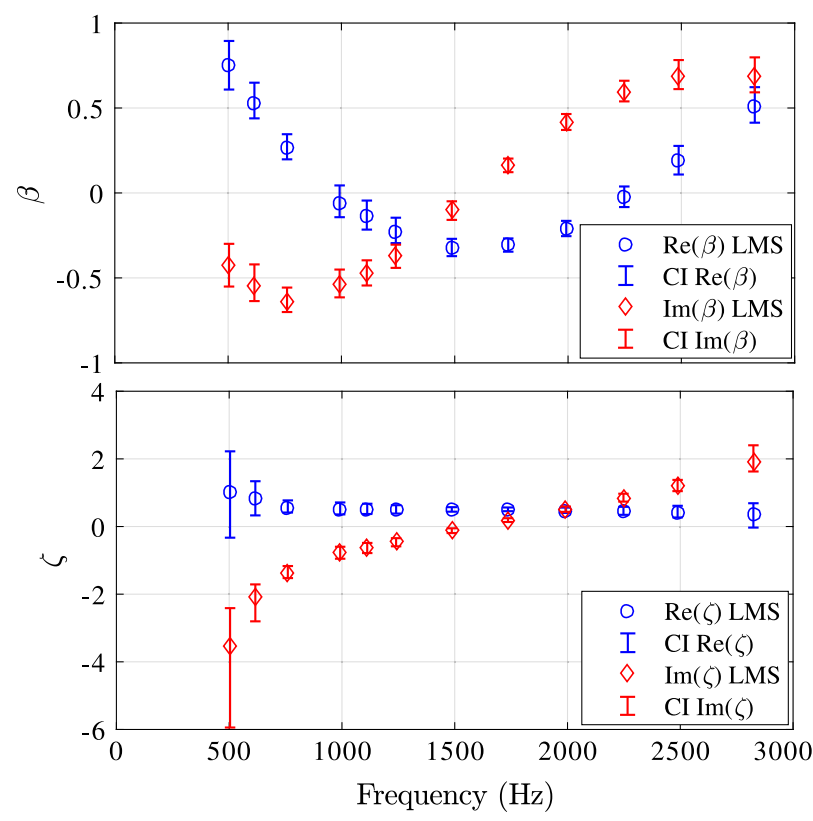

Fig. 9 Comparison between deterministic (LMS) and Bayesian inference results at $M_{b}=0.26$.

involve then only microphone measurements. One of the key strengths of Bayesian inference is its capability to simply describe in a robust way any prior information, and to evaluate the additional knowledge brought by a new observation.

\section{Analysis at the Resonance}

The frequency at which liner resonance occurs (i.e., when the reactance becomes 0 , around $1700 \mathrm{~Hz}$ ) is of particular interest, because this is where the acoustical attenuation is usually maximum in the absence of flow. To investigate the eduction results in more detail at this particular frequency, the analysis performed at resonance in Sec. III.C for the NASA sample was conducted again. The posterior $\overline{\mathrm{PDF}}$ are displayed in Fig. 10. At the examination of the joint PDFs between parameters (off-diagonal elements of Fig. 10), it appears that no strong correlations are present between the parameters, contrasting the results obtained previously for the NASA sample when using microphone measurements on the wall opposite the liner. Setting the numerical SPL at a fixed wrong value would result in a smaller variation of the educed impedance, compared to a case, in which strong correlations would have been present. These correlations are not related to the possible nonlinear behavior of the liner with respect to the source level, which can be quite significant for conventional SDOF liners. These correlations are purely numerical and model based, and depend strongly on the set of measurement points. 


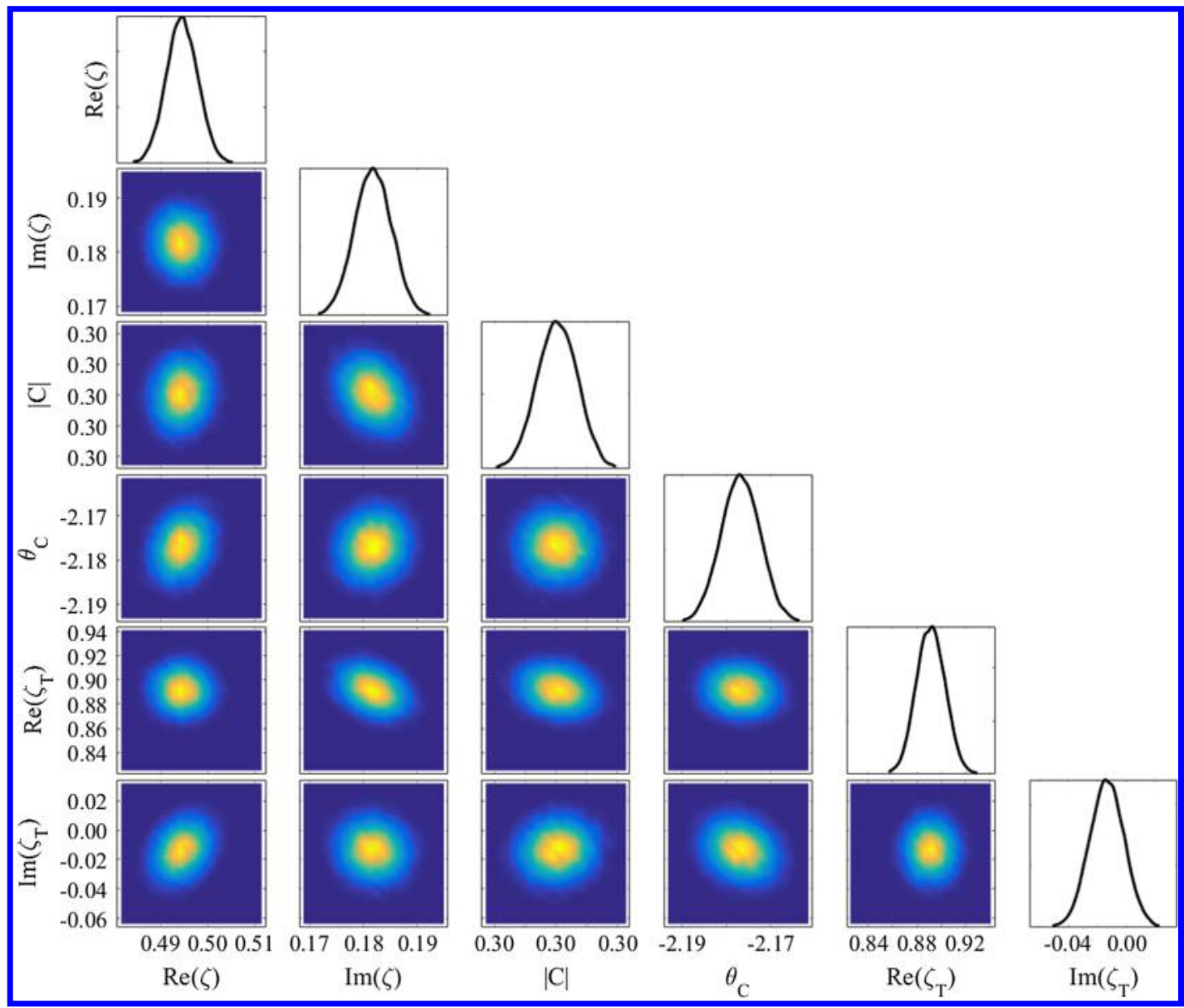

Fig. 10 Inferred parameters for ONERA SDOF material at $M_{b}=0.26$ and $f=1700 \mathrm{~Hz}$

\section{Conclusions}

This paper has presented a statistical inference method based on Bayes's theorem to educe the impedance of a liner in the presence of a grazing flow. The termination impedance and the source amplitude and phase have been inferred as well. The direct problem of wave propagation in a flow duct subject to a grazing flow was represented by the 2-D LEEs, and was solved using a discontinuous Galerkin scheme. An evolutionary Markov chain Monte Carlo technique has been coupled to a surrogate model based on the method of snapshots to efficiently obtain the posterior PDF of the impedance at each frequency of interest. The inference results were first validated against NASA benchmark data, showing a close agreement between educed impedances. Finally, the approach was extended to ONERA's B2A aeroacoustic bench measurement capabilities, where the two components of the acoustic-velocity fields above the liner have been used to feed the inference process. A close match between classical and statistical inference methods was observed. The additional advantage of the Bayesian inference strategy is that it yields the posterior probability density of the impedance at each frequency. In addition, a detailed analysis has been conducted at the liner resonance for the two inferences. This showed that correlations between the educed parameters were absent in the laser Doppler velocimetry (LDV)-based eduction, whereas strong correlations were evidenced in the microphone-based eduction. It is proposed that the larger amount of data obtained through the LDV measurements allows for decorrelated identified parameters.

\section{Acknowledgments}

The authors wish to thank Airbus for the single degree-of-freedom sample manufacturing. A part of this work has received funding from the Clean Sky 2 Joint Undertaking under the European Union's Horizon 2020 Research and Innovation programme under grant agreement number 681856-ASPIRE. The authors warmly thank Mike Jones for providing the data of the NASA Grazing Incidence Tube benchmark published in AIAA 2005-2853 paper. The first author would like to thank the French region Occitanie for its financial support.

\section{References}

[1] Sun, X., Jing, X., Zhang, H., and Shi, Y., "Effect of Grazing-Bias Flow Interaction on Acoustic Impedance of Perforated Plates," Journal of Sound and Vibration, Vol. 254, No. 3, 2002, pp. 557-573. doi:10.1006/jsvi.2001.4110

[2] Zhang, Q., and Bodony, D. J., "Numerical Investigation of a Honeycomb Liner Grazed by Laminar and Turbulent Boundary Layers," Journal of Fluid Mechanics, Vol. 792, April 2016, pp. 936-980. doi:10.1017/jfm.2016.79

[3] Jing, X., Peng, S., and Sun, X., "A Straightforward Method for Wall Impedance Eduction in a Flow Duct," Journal of the Acoustical Society of America, Vol. 124, No. 1, 2008, pp. 227-234. doi: $10.1121 / 1.2932256$

[4] Elnady, T., Bodén, H., and Elhadidi, B., "Validation of an Inverse SemiAnalytical Technique to Educe Liner Impedance," AIAA Journal, Vol. 47, No. 12, 2009, pp. 2836-2844. doi: $10.2514 / 1.41647$

[5] Zhou, L., Boden, H., Lahiri, C., Bake, F., Enghardt, L., BusseGerstengarbe, S., and Elnady, T., "Comparison of Impedance Eduction Results Using Different Methods and Test Rigs," 20th AIAA/CEAS Aeroacoustics Conference, AIAA Paper 2014-2955, 2014. doi:10.2514/6.2014-2955

[6] Jones, M. G., Watson, W. R., and Parrott, T. L., "Benchmark Data for Evaluation of Aeroacoustic Propagation Codes with Grazing flow," AIAA Paper 2005-2853, May 2005. doi:10.2514/6.2005-2853

[7] Eversman, W., and Gallman, J. M., "Impedance Eduction with an Extended Search Procedure," AIAA Journal, Vol. 49, No. 9, 2011, pp. $1960-1970$. doi:10.2514/1.J050831 
[8] Primus, J., Piot, E., and Simon, F., "An Adjoint-Based Method for Liner Impedance Eduction: Validation and Numerical Investigation," Journal of Sound and Vibration, Vol. 332, No. 1, 2013, pp. 58-75. doi:10.1016/j.jsv.2012.07.051

[9] Jones, M., Watson, W., and Nark, D., "Effects of Flow Profile on Educed Acoustic Liner Impedance," 16th AIAA/CEAS Aeroacoustics Conference, AIAA Paper 2010-3763, June 2010. doi:10.2514/6.2010-3763

[10] Aurégan, Y., Leroux, M., and Pagneux, V., "Measurement of Liner Impedance with Flow by an Inverse Method," 10th AIAA/CEAS Aeroacoustics Conference, AIAA Paper 2004-2838, May 2004. doi:10.2514/6.2004-2838

[11] Busse-Gerstengarbe, S., Richter, C., Thiele, F. H., Lahiri, C., Enghardt, L., Roehle, I., Ferrante, P., and Scofano, A., "Impedance Eduction Based on Microphone Measurements of Liners Under Grazing Flow Conditions," AIAA Journal, Vol. 50, No. 4, 2012, pp. 867-879. doi:10.2514/1.J051232

[12] Piot, E., Primus, J., and Simon, F., "Liner Impedance Eduction Technique Based on Velocity Fields," 18th AIAA/CEAS Aeroacoustics Conference (33rd AIAA Aeroacoustics Conference), AIAA Paper 20122198, June 2012. doi: $10.2514 / 6.2012-2198$

[13] Primus, J., Piot, E., Simon, F., Jones, M. G., and Watson, W., "ONERANASA Cooperative Effort on Liner Impedance Eduction," 19th AIAA/ CEAS Aeroacoustics Conference, AIAA Paper 2013-2273, May 2013. doi: $10.2514 / 6.2013-2273$

[14] Brown, M. C., Jones, M. G., and Watson, W. R., "Uncertainty Analysis of the Grazing Flow Impedance Tube," AIAA Paper 2012-2296, June 2012 doi: $10.2514 / 6.2012-2296$

[15] Zhou, L., and Bodén, H., "A Systematic Uncertainty Analysis for Liner Impedance Eduction Technology," Journal of Sound and Vibration, Vol. 356, Nov. 2015, pp. 86-99. doi:10.1016/j.jsv.2015.07.001

[16] Buot de l'Epine, Y., Chazot, J.-D., and Ville, J.-M., "Acoustical Impedance Characterization of Liners Using a Bayesian Approach," Proceedings of Meetings on Acoustics ICA2013, Vol. 19, Acoustical Soc. of America, 2013, Paper 030108. doi: $10.1121 / 1.4800548$

[17] Léon, O., Piot, E., Sebbane, D., and Simon, F., "Measurement of Acoustic Velocity Components in a Turbulent Flow Using LDV and High-Repetition Rate PIV," Experiments in Fluids, Vol. 58, No. 6, 2017, p. 72. doi:10.1007/s00348-017-2348-4

[18] Pascal, L., Piot, E., and Casalis, G., "Discontinuous Galerkin Method for the Computation of Acoustic Modes in Lined Flow Ducts with Rigid Splices," Journal of Sound and Vibration, Vol. 332, No. 13, 2013, pp. 3270-3288. doi:10.1016/j.jsv.2013.01.021

[19] Pascal, L., Piot, E., and Casalis, G., "Discontinuous Galerkin Method for Acoustic Modes Computation in Lined Ducts," 18th AIAA/CEAS Aeroacoustics Conference (33rd AIAA Aeroacoustics Conference), AIAA Paper 2012-2153, June 2012. doi: $10.2514 / 6.2012-2153$

[20] Xiang, N., and Fackler, C., "Objective Bayesian Analysis in Acoustics," Acoustics Today, Vol. 11, No. 2, 2015, pp. 54-61.

[21] Metropolis, N., Rosenbluth, A. W., Rosenbluth, M. N., Teller, A. H., and Teller, E., "Equation of State Calculations by Fast Computing Machines," Journal of Chemical Physics, Vol. 21, No. 6, 1953, pp. 1087-1092. doi:10.1063/1.1699114

[22] Hastings, W. K., "Monte Carlo Sampling Methods Using Markov Chains and Their Applications," Biometrika, Vol. 57, No. 1, 1970, pp. 97-109. doi:10.1093/biomet/57.1.97

[23] Gilks, W. R., Richardson, S., and Spiegelhalter, D., Markov Chain Monte Carlo in Practice, CRC Press, Boca Raton, FL, 1995, Chap. 1. doi: $10.1201 / \mathrm{b} 14835$

[24] Storn, R., and Price, K., "Differential Evolution-A Simple and Efficient Heuristic for Global Optimization over Continuous Spaces," Journal of Global Optimization, Vol. 11, No. 4, 1997, pp. 341-359. doi:10.1023/A:1008202821328
[25] Ter Braak, C. J., "A Markov Chain Monte Carlo Version of the Genetic Algorithm Differential Evolution: Easy Bayesian Computing for Real Parameter Spaces," Statistics and Computing, Vol. 16, No. 3, 2006, pp. 239-249. doi:10.1007/s11222-006-8769-1

[26] Laloy, E., and Vrugt, J. A., "High-Dimensional Posterior Exploration of Hydrologic Models Using Multiple-Try $\operatorname{DREAM}_{(\mathrm{ZS})}$ and HighPerformance Computing," Water Resources Research, Vol. 48, No. 1, 2012.

doi:10.1029/2011WR010608, w01526

[27] Vrugt, J. A., Ter Braak, C., Diks, C., Robinson, B. A., Hyman, J. M., and Higdon, D., "Accelerating Markov Chain Monte Carlo Simulation by Differential Evolution with Self-Adaptive Randomized Subspace Sampling," International Journal of Nonlinear Sciences and Numerical Simulation, Vol. 10, No. 3, 2009, pp. 273-290. doi:10.1515/IJNSNS.2009.10.3.273

[28] Pinnau, R., "Model Reduction via Proper Orthogonal Decomposition," Model Order Reduction: Theory, Research Aspects and Applications, Vol. 13, Springer, Berlin, 2008, pp. 95-109. doi:10.1007/978-3-540-78841-6 5

[29] Cui, T., Marzouk, Y. M., and Willcox, K. E., "Data-Driven Model Reduction for the Bayesian Solution of Inverse Problems," International Journal for Numerical Methods in Engineering, Vol. 102, No. 5, 2015, pp. 966-990. doi: $10.1002 / \mathrm{nme} .4748$

[30] Gelman, A., Carlin, J. B., Stern, H. S., Dunson, D. B., Vehtari, A., and Rubin, D. B., Bayesian Data Analysis, Vol. 2, CRC Press, Boca Raton, FL, 2014, Chap. 1.

[31] Štigler, J., "Analytical Velocity Profile in Tube for Laminar and Turbulent Flow," Engineering Mechanics, Vol. 21, No. 6, 2014, pp. 371-379.

[32] Jing, X., Peng, S., Wang, L., and Sun, X., "Investigation of Straightforward Impedance Eduction in the Presence of Shear Flow," Journal of Sound and Vibration, Vol. 335, Jan. 2015, pp. 89-104. doi:10.1016/j.jsv.2014.08.031

[33] Chung, J. Y., and Blaser, D. A., "Transfer Function Method of Measuring In-Duct Acoustic Properties. I. Theory," Journal of the Acoustical Society of America, Vol. 68, No. 3, 1980, pp. 907-913.

[34] Lavieille, M., "Développement d'une méthode de mesure acoustique en écoulement rapide et chaud par Vélocimétrie Laser Doppler," Ph.D. Thesis, Institut Supérieur de l'Aéronautique et de l'Espace, Université de Toulouse, Toulouse, France, Jan. 2008.

[35] Minotti, A., Simon, F., and Gantié, F., "Characterization of an Acoustic Liner by Means of Laser Doppler Velocimetry in a Subsonic Flow," Aerospace Science and Technology, Vol. 12, No. 5, 2008, pp. 398-407. doi:10.1016/j.ast.2007.09.007

[36] Micheli, F., Lavieille, M., and Millan, P., "ASSA, un outil de référence pour le traitement du signal en vélocimétrie laser," Congrès Francophone de Techniques Laser, Toulouse, France, Sept. 2006.

[37] Boden, H., and Abom, M., "Influence of Errors on the Two Microphones Method for Measuring Acoustics Properties in Ducts," Journal of the Acoustical Society of America, Vol. 79, No. 2, 1986, pp. 541-549. doi:10.1121/1.393542

[38] Nance, D., and Ahuja, K. K., "Limitations of the Three-Microphone Signal Enhancement Technique," Proceedings of the 45th AIAA Aerospace Sciences Conference, AIAA Paper 2007-0441, Jan. 2007. doi:10.2514/6.2007-441

[39] Lavieille, M., Simon, F., and Micheli, F., "Measurement of Acoustic Quantity Fields in Duct Flow by Laser Doppler Velocimetry," Proceedings of the 12th AIAA/CEAS Aeroacoustics Conference, AIAA Paper 2006-2550, May 2006. doi:10.2514/6.2006-2550

[40] Betgen, B., Galland, M., Piot, E., and Simon, F., "Implementation and Non-Intrusive Characterization of a Hybrid Active-Passive Liner with Grazing Flow," Applied Acoustics, Vol. 73, Nos. 6-7, June-July 2012, pp. 624-638. doi:10.1016/j.apacoust.2012.01.008

[41] Efron, B., and Tibshirani, R. J., An Introduction to the Bootstrap, CRC Press, Boca Raton, FL, 1994, Chap. 6. 